\title{
Challenges experienced by cancer patients receiving treatment at Oshakati oncology clinic, Oshana region, Namibia
}

\author{
Ester Mulenga ${ }^{1 *}$, Elise Mutanga ${ }^{2}$ \\ ${ }^{1}$ University of Namibia, Faculty of Health Sciences and Veterinary medicine \\ ${ }^{2}$ Ministry of Health and Social Services, Namibia \\ *Corresponding author E-mail: emulenga@unam.na
}

\begin{abstract}
People living with cancer encounter different problems; some are related to their diagnoses and treatment. The purpose of the study was to explore and describe the challenges experienced by cancer patients receiving treatment at Oshakati oncology clinic in Namibia. A qualitative, exploratory and descriptive study was conducted using purposive sampling and twelve patients diagnosed with different types of cancer were interviewed using unstructured individual in-depth interview. Data were transcribed verbatim and Tesch `s method was used to analyze the data.

The study revealed multifaceted challenges which include physical constraints such as physical ailments, long distance to health facilities and excessive travelling; financial and resource constraints as well as other challenges such as negative attitudes of health workers, poor communication and inadequate provision of information to patients. The study concluded that understanding those challenges will assist the government, private sectors, non- governmental organization and health workers to come up with intervention strategies to support cancer patients effectively. The study recommends the need to decentralize oncology services and to provide in-service training to the health workers working with patients living with cancer.
\end{abstract}

Keywords: Cancer Patient; Challenges; Oncology Clinic; Receiving; Treatment.

\section{Introduction}

Cancer is one of the global health problems. People living with different types of cancer experienced some challenges which prevent them from living a normal life. In Namibia, about 400 men died due to Cancer of which $24.6 \%$ was due to prostate cancer, $9.1 \%$ and $7.7 \%$ due to oropharynx and trachea, bronchus and lungs cancer respectively (World Health Organization (WHO, 2014). Women in Namibia are also affected by different types of cancer. About 300 deaths occurred among women because of cancer whereby $22.3 \%$ was due to breast cancer, while $16.2 \%$ and $7 \%$ were due to cervix uteri and colorectum cancer respectively (WHO, 2014).

Some authors have indicated that people living with cancer are faced with many problems which include financial problem, limited availability of treatments, transportation barriers and inability to do some the work (Miller, 2008; Butterfield, 2017). Some of these problems make people living with cancer vulnerable and dependent on family members and other caretakers. According to Cancer Association of Namibia (2013), there are six common types of cancer namely: skin, prostate, breast, cervical and colorectal cancer as well as Kaposi sarcoma. About 570 men and women are infected by skin cancer; 293 women and 7 men by breast cancer, 334 men by prostate cancer, 264 women by cervical cancer, 260 and 131 men and women by Kaposi sarcoma and colorectal cancer respectively (Cancer Association of Namibia, 2013.)

There are only few public oncology health facilities in Namibia which cater for all patients infected by different types of cancer. One of such facility is situated at the north western part of the country which caters for few selected services such as giving some medications for patients who comes for follow up. The other facility is in the central part of the country which is equipped with all the services available in Namibia for cancer treatment. In case the cancer patient needs treatment such as radiation and chemotherapy, such patient has to travel the central oncology health facility. This means that patients have to travel long distance of more than $500 \mathrm{~km}$ to access such service. As a result, some patients miss their follow up. Despite such situation, little was known about other challenges faced by people living with cancer. Hence, the study revealed that people living with cancer experienced financial problems, limited resources, negative attitudes of health workers, inadequate provision of information by health workers as well as treatment side effects. This article will be structured as follow: firstly, the challenges will be reviewed in relation to the literature, subsequently; the methodology used to explore and describe the challenges will be explained, followed by the results and discussion. The article will end with conclusion and recommendations.

\subsection{Physical related problems affecting people living with cancer}

Cancer patients experience different problems including physical related challenges. According to Edwards \& Greef (2017), Cancer patients in South Africa reported physical challenges such as pain and other symptoms, quality of life issues and poor services delivery as well as long distance to oncology facilities. These authors further indicated that some cancer patients have to travel about 50 to 1045 
kilometers to reach treatment centres and they experience discomfort of long journey (Edwards \& Greef, 2017). It was also found that cancer patients have to endure physical ailments such as fatigue, nausea and vomiting and sleeping problems (The effect of cancer, n.d). Edwards \& Greef (2017), Walubita et al. (2018) stated that cancer patients have to endure long waiting time for transport and also long duration of hospitalisation which affect them in performing some of their responsibilities. Livingston (2012) has alluded that cancer patients find themselves traveling a lot between their homes and hospitals for their medical checkups.

\subsection{Financial problems and poor resources}

Some studies have indicated that people living with cancer experience financial challenges. According to Edwards \& Greef (2017), some cancer patients live in poor households and they are the only breadwinners, some have low income or small state grants and they are not be able to cater for their treatment costs. These authors furthermore stated that some cancer patients become unemployed due to illness and this makes it difficult to access financial support and medical aid. Some researchers indicated that cancer patients face shortage of money and this make it difficult to pay for their transport and food cost. (Walubita et al. 2018). According to Owenga and Nyambedha (2018) some cancer patients cannot afford to pay for their cancer treatment due to financial problems. Hansen (2012 has alluded that even family members of cancer patients are financially affected as children are less likely to stay in school due to financial constraints.

According to Yabrof et al. (2008) some people living with cancer find themselves borrowing money or going in debts, filing bankruptcy and unable to cover their medical expenses due to cancer. While some sell out their properties in order to pay for their children school fees due to loss of jobs.

Besides financial challenges, some cancer patients experienced resource challenges such as lack of medications in public health facilities, broken equipment, testing delays, referral backlogs and dirty health facilities which impacted their condition negatively (Edwards \& Greef, 2017). According to Ahmadiani \& Nikafar (2015), one of the major problems with cancer in India is lack of medications in hospitals and patients cannot afford to buy them from private pharmacy as they are very expensive. Ramos et al. (2017) indicated that facilities had inadequate resources and supplies for managing cancer and there are lack of specialized diagnostic machines and oncologist in some health facilities. According to Zheng et al. (2017) health care providers were forced to refer cancer patients for treatment to other facilities far away such as nearby countries due to resources challenges. Edwards \& Greef (2017), Walubita et al. (2018) stated that some cancer patients have to overnight in chairs as there were limited spaces and beds and some patients have to sleep on floor while waiting for transport.

\subsection{Other challenges experienced by people living with cancer}

Cancer patients are also confronted with other challenges such as poor communication, language barriers, limited and inadequate provision of information as well as negative attitudes of health workers toward cancer patients. Edwards \& Greef (2017) pointed out that cancer patients in South Africa had little information about treatment and support services. These authors furthermore stated that there was poor information about treatment options and lack of patient centered care. Whitney et al. (2016) discovered that information challenges led to inadequate understanding of early signs of cancer and limited understanding about treatment side effects. Chou et al (2016) identified poor communication and language barriers as one of the challenges facing cancer patients. As a result, these patients were not able to understand information related to available resources and do not know the questions they need to ask and discuss with the health workers. According to Walubita et al. (2018) poor communication between health workers and patients has hindered the provision of care to cancer patients. Some patients do not understand English and they can only speak local language which may be different from the language spoken by health workers.

These authors furthermore alluded that some health workers have poor attitudes toward cancer patients and they do not pay adequate attention to such patients. According to Miller (2008), some nurses in California did not like to work with cancer patients and they prefer such patients to be taken care by hospice centres. On contrary a study conducted in Botswana indicated that nurses who are working at palliative departments were very polite and kind (Butterfield et al. 2017).

\section{Methods}

A qualitative, descriptive and exploratory design was utilised to obtain in-depth understanding on the challenges experienced by cancer patients (Creswell, 2014). The population for this study was all patients diagnosed with any type of cancer and come for follow up at Intermediate Hospital Oshakati, Oncology clinic.

\subsection{Sample and sampling}

The study utilised purposive sampling to obtain information from participants who have experienced the phenomenon. Inclusion criteria included: patients diagnosed with any type of cancer for one year or more and such patients should be older than 18 years and mentally stable. A total number of 12 patients were interviewed and data saturation was reached.

\subsection{Data collection method}

Data was collected using unstructured individual in-depth interview. The interview was conducted in local language which is Oshiwambo and translated in English. Unstructured interview assisted participants to relate their challenges freely. The interviews were audio recorded with the permission of participants obtained through informed consent. Field notes were also taken to capture non-verbal communication which cannot recorded. Data was collected using interview guide. The main question which was posed to every patient was" what are the challenges you have experienced since you were diagnosed with cancer?” Probing questions were asked depending on participants` responses.

\subsection{Data analysis}

Data was analyzed by adopting Tesch steps of open coding in which transcripts were read to get sense of the content. Emerging ideas were written and organized into distinctive topics and each topic was allocated a code. Topics were then grouped and turned into themes. 


\subsection{Ethical clearance}

The study was approved by the Ministry of Health and Social Services Ethical committee (Ref No. EM 2018). Data was collected after obtaining written informed consent from the participants and audio recorded with the permission of the participants

\section{Results}

\subsection{Physical challenges experienced by cancer patients}

Participants in this study experienced physical challenges which included physical ailments which hinder their performance of daily living activities. This was evident as follow:

Since my hand was cut off; I was never been able to work for myself until now. Every time I just feel dizzy or I am just sweating. I really can't do anything anymore. (P\# 3).

I am just having muscle cramps and my feet are also facing inside now. So as for me I don't see good health within myself anymore". (P\#1)

Participants also indicated that they experienced excessive travelling and long distance to health facilities which make it difficult to access health services since most of them live very far from oncology health services.

"I go to Windhoek (hospital about 700km from where the study has taken place) after every one month depending on the condition of the wound because it can be that I will go today I might be required go back again after one month or sooner than that." (P\#1).

I used to go to Windhoek for radiation and sometimes I can even stay there for the whole week or two weeks. The distance is very long by time you reach Windhoek you will be very tired or even very sick". (P\#10).

\subsection{Financial and resources challenges}

Participants in this study also experienced financial constraints which made it difficult to pay the transport costs. This was expressed as follow:

My husband is dead and now I do not even have money to pay for my transport cost." (P\# 3)

The last time I was going for radiation to Windhoek, I have to take my own transport because I was the only one and it is costly.” (P\# 4)

One will say it is very expensive if you add up the transport costs, but the truth is it is very expensive to come here because is not every one can afford to come here to look for treatment." (P\# 5)

Financial challenges also lead to children of people living with cancer not to continue with their education.

Even my kids ever since they wrote grade twelve, they never continued because there is no money. There was a time I received money from social security but then I just used to pay my water bill”. (P\# 6)

Participants in this study also indicated that they were faced with resource related challenges which affect their treatment negatively as there are time when medications are not available in public health facilities.

Sometimes we don't have medications; we have to go to the private pharmacy to buy medication. so sometimes you can come to the hospital and you are told there are no medications and you should go to the private pharmacy (P\# 4).

Since I was discharged I spent three (3) days without wound dressing, apparently there is no medications for doing dressing. Like you see here were I fold, it's just myself who did it there is no betadine medication at health centre". (P\# 2)

Participants also experience poor sleeping and toilet facilities as well as lack of accommodation when they go for their follow up.

I sleep in the hospital but the problem sometimes there are no beds, so we will be sleeping on the floor and I am old so it's not easy for me. The toilets in the hospital are very dirty, they are not cleaned at all to be used by the patients". (P\# 8)

I sleep in the guest house that I pay with my own money because I don't have accommodation in the hospital, so I just have to look where to sleep over night". (P\# 11)

\subsection{Other challenges experiences by cancer patients}

Participants experiences other challenges related to poor communication, inadequate provision of information and negative attitudes of hospital workers. This was evident as follow:

It happened one day when I went to bath and I was just shocked to see my hairs were falling off in the bathroom. I called the doctor, then I asked him why my hairs fell off then, he just asked me what I have signed for, then he told me that I have cancer and I have to be injected." (P\# 7)

Young nurses in the hospital do misbehave, for instance, sometimes you talk to them in Oshiwambo (a local language) and then they will reply to you in English, while I don't even know English at all." (P\# 2)

Those that serve us with food they also don't respect us. It happened when one day I was given tea and a folk to steer with but when I asked the spoon they answered badly, apparently I should go to the private hospital if I want to use a spoon.” (P\# 12)

\section{Discussion}

It is evident from the study that patients diagnosed with cancer face different challenges which affect their quality of life. They encountered physical ailments which include amputation of body parts and permanent disfigurements which result in these patients not able to perform any duties as reported by Edwards \&Greef, 2017). Findings from this study indicated that cancer patients travel long distance as some of them have to cover a distance of more than $700 \mathrm{~km}$ to access oncology services and these patients have to endure the discomfort of long travel as some of them are elderly as reported by Walubita et al. (2018). It is also evident from this study that cancer patients have to travel many times to visit health facilities for their treatments as some of them go for follow up on a monthly basis as reported by Livingston (2012).

The study also revealed that patients living with cancer experience financial challenges which make it difficult to pay transport cost and school fees for their children as stated by Walubita et al., Yabrof et al. 2008). Findings from this study indicated that patients diagnosed with cancer encountered resources related challenges which include lack of medications in public health facilities, lack of beds and dirty toilets. Some patients have to sleep on the floor or book private accommodations as it was stated by Edwards \& Greef (2017). 
Some patients have to buy medications from private pharmacy which is very expensive as reported by Ahmadiani \& Nikafar (2015). One of the major concerns identified in this study is that patients are not provided with adequate information which enable them to make informed decision as some patients signed consent without knowing the diagnosis and the consequences of the treatment they are receiving. The study also revealed that health workers display negative attitudes toward patients with cancer as some health workers are rude and they communicate in the language the patients do not understand as reported by Walubita et al. (2018). It is therefore necessary to address those challenges to ease the burden of the patients diagnosed with cancer.

\section{Conclusion and recommendations}

Some salient challenges undermine the quality of life of cancer patients. The results from this study showed that patients have difficulties in accessing oncology services due to long distance to health facilities and excessive travelling. They also encounter problem related to paying of transport cost and school fees due to financial constraints and when they go to the hospital for treatment and follow up they are challenged by lack of resources, inadequate provision of information and poor attitudes of health workers. If these challenges are tackled, it may result in improved quality of life of cancer patients. Hence, the study recommended that oncology health services need to be decentralized to other regions to avoid long travelling; health workers need to be trained on customer care and handling of chronic ill patients and patients living with cancer need to be provided with social grants to enable them to pay for their transport and food costs.

\section{Acknowledgement}

We would like to thank the patients who participated in this study, and we also would like to acknowledge the Ministry of Health and Social services for granting us permission to conduct this study.

\section{References}

[1] Ahmadiani S, \& Nikafar S (2017). Challenges of access to medicine and the responsibility of pharmaceutical companies: A Legal perspective. DARU Journal of Pharmaceutical Sciences, 45(2), 185-192.

[2] Butterfield LH, Kaufman, HL, \& Marincola FM (2017). Cancer immunotherapy principles and practices (1st ed.). Dalas, United State of America: Demos medical. https://doi.org/10.1891/9781617052736.

[3] Chou FY, Kuang LY, Lee J, Yoo GJ, Fung LC (2016). Challenges in cancer self-management of patients with limited English proficiency. AsiaPacific Journal of Oncology. 3(3) https://doi.org/10.4103/2347-5625.189815.

[4] Creswell JK. (2014). Research design: Qualitative, quantitative and mixed methods approach (4th ed.). London: SAGE Publication.

[5] Edwards LB \& Greef LE (2017). Exploring grassroots feedback about cancer challenges in South Africa: A discussion of themes derived from content thematic analysis of 316 photon narratives. Pan African Medical Journal. 28(173). https://doi.org/10.11604/pamj.2017.28.173.11894.

[6] Hansen LA. (2012, Feb 9). Challenges patients face in cancer care. Implications for the health care team. Web exclusive.

[7] Livingston J (2012). Improvising medicine: An African oncology ward in an emerging cancer epidemic (1st ed.). United Kingdom: Duke University Press. https://doi.org/10.1515/9780822395768.

[8] Miller P (2008). Unstoppable attitude: Facing the challenge of Cancer (4th ed.). California: iUniverse.

[9] Owenga AJ \& Nyambedha OE. (2018). Perception of cervical cancer patients on their financial challenges in Western Kenya. BioMed Central Journal, $88(2), 562-680$.

[10] Ramos KE, Hoffmann DA, Gerson LS, Liu H (2017). New opportunities and challenges to defeat cancer stem cells. Health Research Reviews, 39(8), 54-74. https://doi.org/10.1016/j.trecan.2017.08.007.

[11] The effect of cancer on social and emotional wellbeing (n.d). Social and emotional issues related to cancer. Retrieved from www.beyondblue.org.au

[12] Walubita M, Sikateyo B, Zulu JM (2015). Challenges for health care providers, parents and patients who face A childhood cancer diagnosis in Zambia. Health Services Research, 36(1), 56-65.

[13] Whitney RL, Bell JF, \& Joseph JG (2016). Predictors of financial difficulties and work modifications among cancer survivors in the United Nation. Journal of Cancer Survivorship, 10(2), 241-250. https://doi.org/10.1007/s11764-015-0470-y.

[14] Yabroff KR, Dowling EC, \& Ekwueme DU (2008). Financial hardship associated with Cancer in the United States: Finding from a population-based sample of adult cancer survivors. Journal of Clinical Oncology, 15(1), 78-84.

[15] Zheng P, Li J, \& Kros MJ (2017) Breakthroughs in modern cancer therapy and elusive cardiotoxicity: Critical research practice gaps, challenges, and insights. Medical Research Reviews, 31(7), 125-137. 